\title{
Décadrages Décadrages
}

cınéma, a travers champs Cinéma, à travers champs

$1-2 \mid 2003$

Le hors-champ

\section{Plans de chutes}

\section{Denis Martin}

\section{OpenEdition}

Journals

Édition électronique

URL : http://journals.openedition.org/decadrages/594

DOI : $10.4000 /$ decadrages.594

ISSN : 2297-5977

\section{Éditeur}

Association Décadrages

\section{Édition imprimée}

Date de publication : 1 octobre 2003

Pagination : 126-131

ISSN : 2235-7823

\section{Référence électronique}

Denis Martin, «Plans de chutes », Décadrages [En ligne], 1-2 | 2003, mis en ligne le 26 avril 2013, consulté le 21 avril 2019. URL : http://journals.openedition.org/decadrages/594; DOI : 10.4000/ decadrages.594 


\section{Plans de chutes}

par Denis Martin

En premier lieu j'avais choisi celui avancé par ces mètres d'images, là où des corps «il en pleuvait». Ils se lançaient, étaient projetés en une chute, qui ne se décidait pas à en finir. Alors je les ai imaginés : toujours collés à l'ascension inversée, suspendus à la vitesse immobile, ne daignant pas (de là leur beauté ?) s'écraser, justement. Je voulais décrire précisément leur visage de suspens, leur parcours sans espace avec beaucoup de temps, la gesticulation incongrue, et ce seul arrêt - qui n'en était pas un. Et j'imaginais cette scène où soudainement: on assisterait à une véritable pluie des corps; à la formation d'une image-sol qui les accueillerait.

Alors dans l'attente de celui qui (prenant sa caméra) permettrait à tous ces corps d'enfin arriver, ces quelques mots:

Depuis quand ne sautent-ils que dans l'image?

(De là, cette assurance: ne jamais rien faire d'autre que tomber.)

"Ils auront beau traverser le plan ils n'en sortiront pas».

Le plan de la chute d'un seul point une ligne.

On passe au sol sans eux.

Pour ceux qui tombent le sol n'existe (n'existera) pas.

On lèverait la tête on les verrait en une suspension immobile de leur chute.

On pourrait recommencer simplement : la chute n'a pas de hors; elle est (quand elle devient) ce qui passe en son champ.

Le sol n'appartient pas à la chute, n'est même pas une de ses bornes. Le sol, corps étranger, comme hors du champ de la chute. Il n'est pas le 
seul. La chute ne souffre pas de hors-champ.

La chute est le lieu immobile (et) changeant d'elle-même.

La chute n'a pas de vitesse. Elle nous contraint à l'inventer pour elle.

Je serai sûrement d'accord avec celui qui nous dira - «on ne chute jamais vraiment».

«On ne tombe pas dans la chute. On n'y arrive pas.»

Quand elle s'en prend à nous, on comprend que la chute ne souffre pas de sol.

(Il faut bien accuser le coup: nous sommes sans fond.)

On continue: la chute n'expose rien d'autre qu'elle-même. Le sol ne ressort, n'est pas du ressort de son champ.

La chute ne peut qu'être rêvée «il n'y a pas de sol lorsque l'on tombe en nous-mêmes». Ceux qui se jettent ne tendent qu’à éveiller son idée.

Ailleurs, j'avais cru apercevoir «ce moment où, dans la chute, rien ne tombe». Aujourd'hui, doué d'une lucidité aléatoire, je poserais plutôt: voir ce moment, dans la chute où rien ne tombe.

En plus,

Un temps mort, l'espace d'où ils tombent; ou: ils tombent dans le temps, morts, défaits de tous espaces.

Ou alors - l'image de nous qui s'ouvre, s'offre à nous, immobile(s).

Et on peut voir à l'œuvre la puissance du défilement: monstration du suspens.

Puis la vitesse de l'effilement, le délitement en absence d'espace, l'éclatement sans gravité qui nous emportent (vers elle). 
Depuis longtemps, assis dans le noir: on apprécie différentes chutes - de celles du monde.

Puis on dira - pour parler par image (en faire une reste souvent la solution) - ... 24 chutes par seconde. Je les ai toutes vues. Aucune ne s'est arrêtée...

L'image la première, aura compris que la chute n'est que suspension (d'elle-même, en elle).

Avoir une image arrêtée de la chute c'est voir, depuis ce lieu seul qui nous permet, l'image d'une chute arrêtée. On le sait bien, mais on le redit encore autrement: la chute, elle ne tombe pas.

La chute est une certaine forme de (la) réalisation de l'image fixe. (J'ose : l'image est la chute idéale.)

Quelque part, on nous dit - Il y a un espace entre deux images. C'est l'instant pour la chute!... L'espace du temps où son mouvement (et d'autres avec elle) s'élabore. La trace en absence de sa présence. Lieu de la discrétion essentielle.

Confiscation de la chute vol de la chute chute de l'envol Voler, pour nous, ce n'est qu'atteindre artificiellement le lieu de la chute.

On ne peut s'y maintenir que si on l'atteint. Mais sa vérité est dans notre atteinte, lorsque ce lieu-lien étrange d'espace et de temps, se porte à nous.

Ce qui nous manque, essentiellement, c'est l'envol.

Il n'y a qu'une seule chute au monde. Nous la connaissons intimement.

Partout un seul lieu de la chute. Ce lieu arrive sur nous dès que l'on saute. 
L'instant d'après être toujours dans la chute. L'instant d'après aussi.

Il n'y a donc pas de plan - on avancerait un axe: celui de la marche des choses - qu'on suppose souvent vertical.

Et la chute qui devient son image.

Plan de chute, plan de coupe : déchets dans l'ordre du monde - découverte des mobiles de l'immobile.

Plans de chute, chute des plans - l'horizontal en perte de sens. Il disparaît.

Dire de la chute qu'elle est défense du suspens.

La chute comme interdite: elle ne se résout pas au cri qui parfois la porte.

(D’aucuns voudraient mesurer la chute à la distance de son cri.)

Je croyais qu'il fallait comprendre, dans l'ensemble de la chute, le corps de ceux qui ne sautent pas. J'en suis certain maintenant. (Du reste, nous ne sommes pas d'ailleurs.)

Des corps qui ne voudraient œuvrer que dans l'oubli de leurs chutes.

La chute c'est avant tout la disparition du corps en chute.

En face d'elle, on peut se demander si un corps jamais n'atterrit.

Et l'image (qu'on a cru voir plus haut) qui tomberait avec ce corps.

On ne sait jamais vraiment quand un corps tombe. 
On ne sait jamais vraiment, quand un corps tombe, saisir autre chose que l'instant où il finit de tomber.

Alors que nous savons qu'on entre dans la chute pour ne plus en sortir.

Ce n'est pas le corps qui, mais la chute quelconque.

Au corps on préfère l'image de la chute (dans l'image se cache la chute). «Mon corps est objet de chute, ma chute objet de corps».

Le corps, une définition de la chute.

Champs de la chute: le corps en trombe, évoque musical les notes graves, gravitations $\mathrm{du}$ "tombe... ne te relève jamais... descend... on est mieux en bas, même s'il n’y en a pas...».

L'instant vrai de la chute, lorsqu'il n'y a plus de corps qu'elle-même.

Corps, traversée de champ ; ou corps traversé de champ (?).

Où le corps devient l'objet même de la traversée qu'il décrit; qu'il suit, en la précédant; qu'il accompagne, en la délaissant. Pour le corps: le trajet, le transport en la chute le nomme autre en le faisant disparaître. (Il faudrait nommer les objets qui tombent autrement. Idem des choses cassées.)

Dire la chute: la voir devant nous. (Et ne dit-on pas parfois devant pour dans?)

La chute c'est l'œilleton qui nous discerne. Nous, des deux côtés de la porte.

Hors la chute pas de stable - juste une autre chute.

La chute pourrait passer pour un défi désinvolte, alors que sans cause (presque sans conséquence), elle est une des conditions.

La chute n'est pas dans la complexité de la marche; de l'arrêt; de la course, même folle, effrénée. Elle est, simplement : la traversée immobile d'un espace en suspens. 
Autre définition de la chute: qu'elle s'arrête une fois, définitivement.

La chute nous rend, après nous avoir pris, inapparents.

La chute est là, à nos pieds.

Au-delà de la chute il y a la ténacité des objets du monde. 